\title{
INFARTO AGUDO DO MIOCARDIO: ASSITÊNCIA AO PACIENTE PÓS-INFARTO INTERNADO EM UNIDADE DE TERAPIA INTENSIVA
}

\author{
ACUTE MYOCARDIAL INFARCTION: ASSISTANCE TO HOSPITAL POST-INFARCTION \\ PATIENTS IN INTENSIVE CARE UNIT
}

Iran Miranda Silva ${ }^{1}$, Marcos Gontijo da Silva².

\section{RESUMO}

Introdução: O Infarto Agudo do Miocárdio (IAM) se dá por uma oclusão da artéria coronária, através da formação de um coágulo ou placa de ateroma, diminuindo o fluxo sanguíneo e levando parte do miocárdio a um processo de necrose o que desencadeia em um grande número de óbitos na população mundial.

Objetivo: Realizar uma revisão sistemática de literatura sobre o infarto agudo do miocárdio, enfatizando a assistência ao paciente pós-infarto internado em Unidade de Terapia Intensiva.

Metodologia: Foi realizada uma busca em portais científicos como PubMed e SciELO utilizando como como descritores os termos "Infarto Agudo do Miocardio" e "UTI".O período de busca foi de 2011 a 2016.

Resultados e discussão: Na Pubmed foram encontrado 151 artigos e na SciELO 126, totalizando 277 artigos. Foram excluídos os artigos que não tratavam sobre assistência ao paciente, restando 26 artigos, estes que comporam esse estudo. Os artigos selecionados foram analisados e os assuntos separados em 09 (nove) tópicos para melhor entendimento do tema.

Considerações finais: foi observada a enfâse das publicações no fato do IAM ser uma patologia de facil diagnóstico, mas que se não tratada a tempo possui um alto indice de mortes nas primeiras horas. A gravidade depende da extensão da região atingida e de todos os fatores de riscos associados.

Descritores: Infarto Agudo do Miocárdio. UTI. Assistência.

\section{ABSTRACT}

Introduction: Acute myocardial infarction (IAM) is given by a coronary artery occlusion, through the formation of a clot or atheroma plaque, decreasing blood flow and taking part of the myocardium to a process of necrosis which triggers in a large number of Deaths in the world population.

Objective: To carry out a systematic review of literature on acute myocardial infarction, emphasizing the assistance to the patient postmyocardial interned in intensive therapy unit.

Methodology: A search was carried out on scientific portals such as PubMed and SciELO using as descriptors the terms "acute infarction of the infarction" and "UTI". The search period was 2011 to 2016.

Results and discussion: In Pubmed were found 151 articles and in SciELO 126, totaling 277 articles. Articles that did not deal with patient care were excluded, leaving 26 articles, the ones that composed this study. The selected articles were analyzed and the subjects separated in 09 (nine) topics for better understanding of the theme.

Final considerations: It was observed the emphasis of the publications in the fact of the IAM to be a pathology of easy diagnosis, but that if not treated in time has a high index of deaths in the first hours. Gravity depends on the extent of the affected region and all associated risk factors.

Descriptors: Acute myocardial infarction. ITU. Assistance.

\section{INTRODUÇAO}

O termo infarto do miocárdio significa basicamente a morte de cardiomiócitos causada por isquemia prolongada. Em geral, essa isquemia é causada por trombose e/ou vaso espasmo sobre uma placa ateroma ótica. $O$ processo migra do subendocárdio para o subepicárdico. A maior parte dos eventos é causada por rotura súbita e formação de trombo sobre placas vulneráveis, inflamadas, ricas em lipídios e com capa fibrosa delgada. Uma porção menor está associada à erosão da placa aterosclerótica ${ }^{1}$.

No Brasil, as doenças cardiovasculares ainda permanecem como a primeira causa de mortalidade proporcional, responsáveis por $29 \%$ dos óbitos em 2010 (Departamento de Informática do Sistema Único de Saúde [Datasus] ${ }^{2}$. O infarto agudo do miocárdio (IAM) é a segunda causa de morte mais frequente (7\%, Datasus 2010), e no sistema público de saúde a mortalidade hospitalar dos pacientes internados por IAM se mantém persistentemente elevada: em média, $16,2 \%$, em $2000,16,1 \%$, em 2005 , e $15,3 \%$, em 2010 , para as internações 
10.18606/2318-1419/amazonia.sci.health.v6n1p12-21 Revista Amazônia Science \& Health.

2018 Jan/Mar
Silva IM, Silva MG.

Infarto Agudo do Miocardio: Assitência ao paciente pós-infarto internado em Unidade de Terapia Intensiva. registradas em todo país (Datasus). A elevada mortalidade no sistema público de saúde brasileiro é atribuída às dificuldades no acesso do paciente com IAM ao tratamento em terapia intensiva, aos métodos de reperfusão e às medidas terapêuticas estabelecidas para o IAM.

Todo procedimento em UTI é considerado essencial ao paciente e por isso é de extrema importância que o mesmo seja realizado precisamente, no entanto, sabe-se que existem algumas técnicas que deixam de ser realizadas, algumas vezes, devido aos atendimentos emergenciais que ocorrem na unidade como parada cardiorrespiratória e intubação ${ }^{3}$.

A assistência de enfermagem em UTI merece uma atenção_especial, pois durante a realização de uma técnica, o cuidar do profissional ao manusear tantos artefatos terapêuticos e diagnósticos pode causar sérios prejuízos para a continuidade da assistência, tais como a saída acidental, obstrução ou posicionamento incorreto de cateteres, cânulas, sondas e drenos ${ }^{3}$.

O prognóstico positivo desses pacientes depende fundamentalmente da agilidade em alcançar um serviço médico e na eficiência desse serviço em obter a reperfusão coronariana o mais rápido possível ${ }^{1}$.

Assim esse trabalho possui como objetivo realizar uma revisão sistemática da literatura sobre o infarto agudo do miocárdio, enfatizando a assistência ao paciente pós-infarto internado em Unidade de Terapia Intensiva.

\section{METODOLOGIA}

Este trabalho é um estudo do tipo revisão de literatura, a qual compilou resultado de pesquisas sobre o tema Infarto agudo do miocárdio, de maneira sistemática e ordenada, contribuindo para o aprofundamento do conhecimento do assunto investigado.

Para realização dessa revisão, inicialmente, foi identificada a questão de pesquisa, que foi "a importância da assitência ao paciente pós-infarto internado em Unidade de Terapia Intensiva" aliada à seleção dos descritores "Infarto Agudo do Miocardio, UTI", e, em seguida, foram estabelecidos os critérios de inclusão e exclusão de artigos (foram usados artigos publicados nos últimos 5 anos). A partir disso, as seguintes etapas foram percorridas durante a elaboração desse estudo: foi realizada a seleção da amostra através da busca nas bases de dados; posteriormente, houve a sumarização das informações extraídas dos artigos selecionados; avaliação dos estudos; interpretação e discussão dos resultados; e a última etapa foi constituída pela apresentação da revisão e síntese do conhecimento.

Para seleção dos artigos, foram utilizadas as seguintes bases de dados: PubMed e SciELO. A busca foi realizada pelo entre os meses de maio e junho de 2016. Os critérios de inclusão dos artigos definidos para esta revisão foram: ter sido publicado nos últimos 5 anos; no idioma português; estar disponível eletronicamente na íntegra; e abordar o Infarto Agudo do Miocardio relacionado a assistência em Unidade de Terapia Intensiva. Os estudos que não se enquadraram nas características citadas foram excluídos desta revisão. Foram utilizados os descritores controlados: "Infarto Agudo do Miocardio" e "UTI".

Os resultados encontrados foram descritos por meio de frequências, apresentados em forma de texto e discutidos segundo a literatura pertinente, possibilitando ao leitor a avaliação da aplicabilidade da revisão integrativa elaborada, a fim de atingir o objetivo desse método.

\section{DESENVOLVIMENTO}

Segundo Pesaro ${ }^{4,}$ existe um padrão dinâmico de trombose e trombólise simultaneamente, associadas à vasoespasmo, o que pode causar obstrução do fluxo intermitente e embolização distal (um dos mecanismos responsáveis pela falência da reperfusão tecidual apesar da obtenção de fluxo na artéria acometida).

Dentro de um espectro de possibilidades relacionadas com o tempo de evolução, o miocárdio sofre progressiva agressão representada pelas áreas de isquemia, lesão e necrose sucessivamente. $\mathrm{Na}$ primeira, predominam distúrbios eletrolíticos, na segunda, alterações morfológicas reversíveis e na última, danos definitivos ${ }^{4}$.

Conforme Pesaro ${ }^{4}$, essas etapas se correlacionam com a diversidade de apresentações clínicas que variam da angina instável e infarto sem supra até o infarto com supra desnível do segmento ST. É por isso que o manejo do infarto é baseado no rápido diagnóstico, na desobstrução imediata da coronária culpada, manutenção do fluxo obtido, profilaxia da embolização distal e reversão de suas complicações potencialmente fatais (arritmias, falência cardíaca e distúrbios mecânicos).

Nas últimas décadas, ocorreu redução importante na taxa de mortalidade por doenças cardiovasculares relacionadas a avanços na prevenção primária e no tratamento da síndrome coronariana aguda (SCA). Embora essa redução seja tendência mundial, é mais pronunciada em países desenvolvidos, nos quais é possível ter acesso, em tempo hábil, ao tratamento adequado, com reperfusão por angioplastia primária ou fibrinólise, terapia antitrombótica dupla e tratamento intensivo ${ }^{2}$.

De acordo com Marcolino ${ }^{2}$, o reconhecimento de que para o tratamento adequado do IAM é necessária a interação de vários setores (comunidade, unidades de pronto atendimento, 
10.18606/2318-1419/amazonia.sci.health.v6n1p12-21 Revista Amazônia Science \& Health.

2018 Jan/Mar
Silva IM, Silva MG.

Infarto Agudo do Miocardio: Assitência ao paciente pós-infarto internado em Unidade de Terapia Intensiva. serviço de transporte de emergência e hospital com serviços de hemodinâmica e de terapia intensiva) induz a criação de sistemas ou Linhas de Cuidado do IAM para otimizar o atendimento ao paciente, desde 0 diagnóstico precoce até o tratamento adequado e em tempo hábil.

\section{Caracterização do IAM}

O infarto agudo do miocárdio (IAM) é o maior problema de saúde pública nos países industrializados. Sua incidência vem aumentando assustadoramente nos países em desenvolvimento ${ }^{6}$.

O estudo do infarto agudo do miocárdio (IAM) é fundamental pela alta prevalência, mortalidade e morbidade da doença. Estudos epidemiológicos revelam taxas de mortalidade geral ao redor de $30 \%$, sendo que metade dos óbitos ocorrem nas primeiras duas horas do evento e 14\% morrem antes de receber atendimento médico. No entanto, os pacientes admitidos nos serviços de emergência precocemente foram os que mais se beneficiaram dos avanços terapêuticos das últimas décadas ${ }^{4}$.

As doenças cardiovasculares continuam sendo a primeira causa de morte no Brasil, responsáveis por quase $32 \%$ de todos os óbitos. Além disso, são a terceira maior causa de internações no país. Entre elas, o infarto agudo do miocárdio ainda é uma das maiores causas de morbidade e mortalidade. Apesar dos avanços terapêuticos das últimas décadas, o infarto ainda apresenta expressivas taxas de mortalidade e grande parte dos pacientes não recebe o tratamento adequado ${ }^{4}$.

De acordo com Pesaro ${ }^{4}$, a mortalidade intrahospitalar, ao redor de $30 \%$ antes de 1960, diminuiu para $16 \%$ com 0 advento das unidades coronarianas. Posteriormente, com o desenvolvimento dos fibrinolíticos e da angioplastia primária, as taxas declinaram até cerca de 6\%-8\% nos primeiros 30 dias após o infarto.

\section{Principais fatores de risco para o IAM}

As doenças cardiovasculares (DCV) são as principais causas de morte e, também, de morbidade e incapacitação entre os países ocidentais desenvolvidos. No âmbito das DCVs, a aterosclerose, tanto a das artérias coronárias como a cerebral, constitui-se, nesses países, em sério problema de saúde pública, assumindo contornos de verdadeira epidemia fatores que predispunham a um maior risco do desenvolvimentoda aterosclerose, entre os quais despontavam como mais importante a faixa etária, o sexo masculino, a hereditariedade, a hipercolesterolemia, a hipertensão arterial sistêmica (HAS) e o diabetes mellitus (DM) ${ }^{7}$.
Os fatores de risco mais freqüentes para doença arterial coronariana foram hipertensão arterial sistêmica. ${ }^{8}$

A partir da identificação desses fatores, iniciou-se persistente trabalho visando sua eliminação ou atenuação, de tal sorte que, na maior parte dos países ocidentais desenvolvidos, vem se percebendo, nas últimas décadas, reversão na tendência de mortalidade por doença arterial coronária $(\mathrm{DAC})^{7}$.

\section{Intervenção no IAM}

O estabelecimento de tratamento eficaz para o infarto agudo do miocárdio (IAM) foi uma das conquistas médicas mais importantes da segunda metade do século XX. A mortalidade hospitalar caiu de alarmantes $30 \%$ a $40 \%$ nas décadas de 1950 e 1960 para menos de 5\% em 2006, em decorrência da introdução de várias estratégias terapêuticas, entre as quais se destacam a instalação das unidades de tratamento intensivo (UTI) cardiovascular, as unidades coronarianas, e a introdução da terapia de reperfusão (química ou percutânea) ${ }^{9}$.

A introdução da intervenção coronariana percutânea (ICP), utilizando um cateter balão, por Andreas Gruentzig, em 1977, revolucionou o tratamento da doença arterial coronariana (DAC). Lesões coronarianas, até então, só foram abordadas como procedimento cirúrgico, envolvendo uma toracotomia, passaram a ser tratadas com um cateter balão levadas até 0 sistema coronariano por simples punção arterial periférica.

A estenose coronariana, complicação comum nos anos iniciais, foi reduzida com o implante dos stents coronarianos utilizados a partir de 1982, procedimento que veio a se tornar_padrão. Os stents não recobertos, inicialmente empregados, foram substituídos, a partir de 2001, por stents farmacológicos, e diluídos primeiro com sirolimus, seguido pelo paclitaxel ${ }^{4}$, e posteriormente por outros diferentes fármacos que, se não aboliram a reestenose e a necessidade de reintervenção, tornaram-na menos frequente ${ }^{11}$.

Segundo Portela ${ }^{10}$ estima-se que atualmente sejam implantados anualmente 2 a 3 milhões de stents no mundo, sendo que aproximadamente $70 \%$ são diluídos com fármacos. A limitação para seu emprego é o custo, principalmente nos países com dificuldades econômicas para manter seus sistemas de saúde.

Os stents não farmacológicos são reembolsáveis, entretanto seu número por paciente é controlado, sendo permitido o implante de até dois stents em $20 \%$ do total de procedimentos por serviço ${ }^{11}$.

Os stents convencionais foram incorporados aos tratamentos fornecidos pelo SUS em dezembro 
10.18606/2318-1419/amazonia.sci.health.v6n1p12-21 Revista Amazônia Science \& Health.

2018 Jan/Mar
Silva IM, Silva MG.

Infarto Agudo do Miocardio: Assitência ao paciente pós-infarto internado em Unidade de Terapia Intensiva. de 1999. Até então, o principal procedimento disponível, era a revascularização cirúrgica ${ }^{11}$.

O tratamento do infarto agudo do miocárdio conta, hoje, com um leque de opções terapêuticas, sempre com o objetivo de reduzir o dano ao ventrlculo esquerdo, Dentre as possibilidades, a revascularização cirúrgica encontra seu lugar, ligado, principalmente, à reperfusão após trombollticos e às complicações da angioplastia ${ }^{12}$.

Inicialmente, a cirurgia era usada como forma de recanalização, sendo realizada de maneira mais precoce após o IAM, com objetivo de redução da área infartada, porém com morbi-mortalidade cirúrgica elevada ${ }^{13}$.

O desenvolvimento da cardiologia intervencionista tem possibilitado que um número maior de pacientes e com maior grau de complexidade seja abordado de forma nãocirúrgica, notadamente na doença arterial coronária. Este fato tem contribuído para o surgimento de novos serviços que realizam tais procedimentos e, como consequência, um volume menor de procedimento por serviço ${ }^{11}$.

\section{Suporte ventilatório ao paciente com IAM}

No período pós-operatório de reperfusão após IAM, o paciente deve ser encaminhado imediatamente à UTI, em ventilação mecânica, com monitorização de pressão venosa central e linha arterial invasiva ${ }^{14}$.

Lima $^{15}$, afirma que a utilização do suporte ventilatório não invasivo vem atuando como coadjuvante da reabilitação cardíaca na tentativa de melhorar a capacidade funcional dos pacientes. No âmbito da reabilitação cardíaca $(\mathrm{RC})$, busca-se o condicionamento do indivíduo, tendo como objetivo a melhoria da capacidade funcional e o retorno às atividades de trabalho e sociais, nas quais o desempenho físico é um fator_limitante. Um dos principais agravos é o désenvolvimento da insuficiência respiratória aguda devido à redução da complacência pulmonar que aumenta o trabalho respiratório. Tal redução é resultado da insuficiência circulatória causada pela disfunção do ventrículo esquerdo (VE), que pode causar prejuízos agudos e/ou crônicos na função respiratória.

Nesse cenário, surge a utilização do suporte ventilatório não invasivo (SVNI) como coadjuvante da reabilitação cardíaca na tentativa de melhorar a capacidade funcional dos_pacientes, uma vez que o SVNI reduz o trabalho respiratório, melhora a oxigenação, aumenta a complacência pulmonar associada à melhora do volume ejetado, devido ao aumento da pressão intratorácica. A utilização do SVNI tem sido uma alternativa na tentativa de aumentar a oxigenação arterial e proporcionar uma melhor tolerância durante o exercício físico devido a sua sensível atuação na interação cardiorrespiratória, proporcionando uma melhor resposta cardíaca e respiratória durante o exercício. Entretanto, são escassos os estudos que levam em consideração a utilização do SVNI associado ou não ao exercício na IC no contexto da $\mathrm{RC}^{15}$.

\section{Terapêutica adjuvante no IAM}

A conduta no infarto agudo do miocárdio (IAM) foi acentuadamente modificada durante a última década com a introdução e a consequente incorporação, na prática clínica, de terapias eficazes e da avaliação de outras novas e promissoras, além do aumento na utilização de técnicas intervencionistas percutâneas ${ }^{16}$.

\section{Betabloqueadores}

O mecanismo pelo qual o betabloqueador reduz eventos cardíacos não está totalmente esclarecido. Sabe-se que ele melhora a relação entre a oferta e o consumo de oxigênio do miocárdio pela diminuição da frequência cardíaca; previne a isquemia; aumenta o enchimento das coronárias na diástole; reduz a área do IAM; estabiliza as placas coronárias; e aumenta o limiar para fibrilação ventricular na presença de isquemia miocárdica ${ }^{17}$.

Os betabloqueadores IV exercem efeito benéfico através de redução do consumo de oxigênio, como antagonistas dos efeitos adversos das catecolaminas, das propriedades antiarrítmicas e da influência favorável na distribuição do fluxo sangüíneo coronário ${ }^{18}$.

O estudo ISIS-1 avaliou o efeito do atenolol intravenoso (IV) iniciado no IAM, ocorrendo moderada redução na mortalidade vascular $(3,9 \%$ vs $4,6 \%$ ) com evidência estatisticamente significativa de efeito benéfico no resultado combinado de morte, parada cardíaca e reinfarto Informações sobre combinação do betabloqueador com terapia trombolítica e aspirina são limitados. No estudo TIMI-Ilb foi comparado o betabloqueador IV imediato VS terapêutica oral tardia em pacientes submetidos à terapia trombolítica. Houve menor incidência de reinfarto, angina recorrente e acidente vascular cerebral (AVC) hemorrágico para aqueles tratados após seis dias no grupo IV imediato. ${ }^{19}$

\section{Inibidores da enzima conversora de angiotensiva}

Segundo Avezum ${ }^{19}$, muitos estudos avaliaram os efeitos dos IECA nas primeiras 24 horas após início do IAM em pacientes não selecionados e, dentre estes, quatro são amplos com poder estatístico adequado: CONSENSUS-II, GISSI-1, ISIS-4, CCS-1. O CONSENSUS-II (enalaprilato IV $V S$ placebo) não demonstrou diferença em termos de mortalidade, com um excesso de hipotensão (12\% no Grupo enalaprilato comparado a 3\% no placebo, $\mathrm{p}<0.001$ ). Os estudos GISSI-3 (lisinopril via 
10.18606/2318-1419/amazonia.sci.health.v6n1p12-21 Revista Amazônia Science \& Health.

2018 Jan/Mar
Silva IM, Silva MG.

Infarto Agudo do Miocardio: Assitência ao paciente pós-infarto internado em Unidade de Terapia Intensiva. oral VS placebo) com redução significativa de $12 \%$ na mortalidade $(2 \mathrm{p}=0,03)$, o CCS- 1 (captopril oral ou placebo) em pacientes com suspeita de IAM, com redução significativa da mortalidade em 4 semanas.

\section{Nitratos}

Os nitratos são vasodilatadores venosos, arteriais e arteriolares, inclusive vasodilatadores coronários $^{18}$.

Em estudos clínicos preliminares, o uso de nitrato IV no IAM mostrava redução da mortalidade de $35 \%(p<0,001)$ e com ouso de nitrato por via oral redução nãosignificante de 21\%. Em conjunto, nitratos por via oral ou IV, mostravam uma redução de $31 \%$ da mortalidade $(p<0,001)$ em 3122 pacientes. Posteriormente, os efeitos dos nitratos no IAM foram avaliados em dois grandes estudos envolvendo mais de 70 mil pacientes. No ISIS-4, que avaliou o uso de isossorbida-5-mononitrato vs placebo em 58.050 pacientes, a mortalidade em 35 dias não diferiu no grupo nitrato (7,34\%) VS controles (7,54\%). O estudo GISSI-3 comparou nitroglicerina $V S$ controle e a mortalidade em 42 dias não apresentou diferença significativa ${ }^{19}$.

De acordo com Avezum ${ }^{19}$ em ambos os estudos o poder para detectar-se qualquer benefício real com a terapia foi reduzida pelo uso precoce e extenso (>50\%) de nitratos nos grupos controles (contaminação). Quando combinamos os dados do ISIS-4, GISSI-3 e de 20 pequenos estudos observase uma redução da mortalidade com nitratos de $5,5 \%(2 p=0,003)$, o que evitaria 3,8 mortes a cada 1.000 pacientes tratados.

\section{Eventos adversos da assitência em UTI}

As instituições de saúde têm como princípio básico no atendimento à clientela o fornecimento de bens e serviços com o mínimo ou a ausência total de riscos e falhas que possam comprometer a segurança do paciente. Entretanto, existem situações que predispõem ao risco de eventos adversos, tais como: avanço tecnológico com incompatibilidade do aperfeiçoamento pessoal necessário, distanciamento das ações próprias de cada profissional, desmotivação, ausência ou limitação da sistematização e documentação do cuidado de enfermagem, delegação de cuidados sem supervisão adequada e sobrecarga de serviço. Os profissionais de saúde, como quaisquer seres humanos, são passíveis de falhas, no entanto, não se identificam os eventos adversos quando os profissionais se solidarizam e mascaram tais situações ${ }^{3}$.

Segundo Beccaria ${ }^{3}$, deve existir um olhar crítico e investigativo sobre as falhas, a fim de apontar lacunas que precisam ser sanadas para beneficiar não só a equipe, mas principalmente o cliente. Vários termos são utilizados como sinônimos de eventos adversos: ocorrências de eventos iatrogênicos; erros de enfermagem; ocorrências, reações; complicações iatrogênicas; iatrogenias; doença iatrogênica e falhas.

Entretanto, todas são definidas como eventos indesejáveis, não intencionais, de natureza danosa ou prejudicial ao paciente, comprometendo sua segurança, consequente ou não de falha do profissional envolvido. O problema dos eventos adversos na UTI está relacionado ao avanço tecnológico e científico caracterizado por diversas aparelhagens e utilização de novas tecnologias diagnósticas e terapêuticas, cuidados específicos, somado ao maior contingente de profissionais envolvidos na assistência.

De acordo com Beccaria ${ }^{3}$, os pacientes de UTI estão mais vulneráveis aos eventos adversos e mais susceptíveis à infecção hospitalar. Um estudo realizado na Faculdade de Medicina da Universidade de Harvard, em Boston, verificou que mais de $20 \%$ dos pacientes admitidos em UTI sofreram algum evento adverso.

A avaliação da assistência é um importante instrumento no controle de processos de trabalho na saúde. A qualidade esperada é a satisfação das expectativas dos pacientes e familiares. Na UTI a expectativa é garantir o melhor resultado dentro das condições clínicas e da gravidade dos pacientes, tendo os menores índices possíveis de complicações decorrentes dos procedimentos realizados.

A notificação e aceitação da existência de eventos adversos facilitam a investigação da qualidade da assistência de enfermagem, pois falhas acontecem e são importantes para a avaliação dos serviços de saúde ${ }^{3}$.

As cirurgias cardíacas são cirurgias de grande porte difundidas mundialmente, dentre elas destaca-se principalmente a revascularização miocárdica (RVM) e as trocas valvares. O paciente submetido à cirurgia cardíaca permanecerá em ventilação mecânica (VM) no pós-operatório imediato até que recobre a total lucidez, em alguns casos por tempo ainda maior, e deverá permanecer no leito por três a seis semanas. Devido à instabilidade hemodinâmica que pode acontecer no pós-operatório imediato, o paciente pode precisar de drogas vasoativas (DVA) e permanecerá na Unidade de Tratamento Intensivo (UTI) enquanto houver necessidade ${ }^{20}$.

As doenças sistêmicas, principalmente as respiratórias e metabólicas, como doença pulmonar obstrutiva crônica (DPOC) e diabetes, podem aumentar o tempo de permanência na UTI e, em alguns casos, aumentar o tempo de $\mathrm{VM}^{20}$.

\section{Assistência de enfermagem ao IAM}

Os profissionais que atuam em unidade de emergência (UE) deveriam cada vez mais receber 
10.18606/2318-1419/amazonia.sci.health.v6n1p12-21 Revista Amazônia Science \& Health.

2018 Jan/Mar
Silva IM, Silva MG.

Infarto Agudo do Miocardio: Assitência ao paciente pós-infarto internado em Unidade de Terapia Intensiva. treinamento específico e aperfeiçoamento técnicocientífico na prática, pois é neste local que a equipe de enfermagem em conjunto com a equipe médica, executam um atendimento sincronizado_ao paciente vítima de trauma ${ }^{21}$

A dor torácica é apontada como uma das principais queixas referidas pelos pacientes que procuramos atendimentos de emergência. De acordo com estudos da Sociedade Brasileira de Cardiologia, a estimativa é de quatro milhões de pessoas atendidas por dor torácica anualmente no Brasil. Cerca de 5 a $15 \%$ dos pacientes que referem dor torácica são diagnosticados com Infarto Agudo do Miocárdio (IAM), ou seja, em dados relativos, 400 mil por ano em nosso país. Considerando que essa dor é o sintoma clássico da Síndrome Coronariana Aguda (SCA), a atenção a ela precisa ser redobrada ${ }^{22}$.

Segundo Vieira ${ }^{22}$, os enfermeiros que atuam em um serviço de emergência, na classificação de risco, precisam estar atentos em vigência da dor torácica, que pode ter em sua origem uma isquemia cardíaca e, diante de sua subjetividade, avaliar e classificar a dor torácica não se constitui em tarefa simples. Portanto, nesse caso, a identificação da gravidade na classificação deve ser rápida e o serviço especializado, visto que o tempo é determinante para o sucesso do atendimento.

Nesse contexto, emergem como tecnologia do cuidado e instrumento para o embasamento da prática de enfermagem, os protocolos assistenciais que vêm ao encontro das necessidades dos enfermeiros para a tomada de decisão durante a classificação de risco. Com relação aos eventos coronarianos, a redução de casos como o IAM na SCA ocorre mediante uso de diretrizes com evidência científica comprovada. $O$ uso de protocolos clínicos é útil para otimizar a qualidade do atendimento ${ }^{22}$.

Ainda de acordo com Vieira ${ }^{22}$, o Acolhimento com Classificação de Risco (ACR) surgiu com a finalidade de melhor coordenar a ordem do atendimento, excluindo a ordem de chegada e incluindo a classificação da gravidade ou fatores de risco associados que predisponham a um possível risco ameaçador à vida. Para a classificação de risco é necessário o trabalho de um profissional de enfermagem de nível superior munido de um instrumento que fundamente a condução_do caso e avalie sua gravidade ou seu potencial de agravamento do caso. Os protocolos assistenciais sistematizam a ação do profissional, além de serem fundamentais para a efetiva classificação de risco e avaliação da vulnerabilidade do paciente.

A proporção de enfermeiros por leito, por plantão, é de 1:10, e de auxiliares/técnicos é de 1:2. Os auxiliares/técnicos de enfermagem prestam assistência integral ao paciente norteada pela prescrição de enfermagem e estão sob supervisão e orientação do enfermeiro. O treinamento em serviço é realizado sempre que necessário em conjunto com o Centro de Educação Permanente da instituição ${ }^{3}$.

\section{Manual de orientações ao IAM}

\section{Atividade Sexual}

A atividade sexual pode ser iniciada em torno de 30 dias após o IAM. Deve-se evitar o ato sexual após refeições abundantes e ingestão de bebida alcoólica (aconselha-se aguardar três horas). Evitar relações sexuais em ambientes com excesso de calor ou frio, quando fadigado ou cansado. $\mathrm{Na}$ vigência de dor anginosa (dor no peito) durante o ato sexual, fazer uso de vasodilatador coronariano conforme orientação médica ${ }^{1}$.

\section{Exercícios físicos}

É importante o retorno lento e gradual às atividades físicas domiciliares, pois o processo de reparação do coração se completa em tomo de 60 a 90 dias. Aconselha-se caminhar diariamente em lugares planos e calmos, aumentando gradativamente 0 percurso (iniciar caminhando cerca de 300 a 400 metros) conforme sua tolerância. Também recomenda-se evitar as caminhadas quando cansado, após refeições e em ambientes conturbados. Recomenda-se que se evite carregar peso até 68 semanas após IAM $^{1}$ (Tabela 1).

\section{Tabela 1. Perfil do paciente infartado ${ }^{5}$}

\begin{tabular}{|c|c|c|}
\hline Semana & $\begin{array}{c}\text { Duração em } \\
\text { minutos }\end{array}$ & Distância em metros \\
\hline $1^{\mathrm{a}}$ a $3^{\mathrm{a}}$ & - & Ativ. físicas no hospital \\
\hline $4^{\underline{a}}$ & 5 minutos & $400-1$ vez ao dia \\
\hline $5^{\mathrm{a}}$ & 5 minutos & $400-2$ vezes ao dia \\
\hline $6^{\mathrm{a}}$ & 10 minutos & $800-1$ vez ao dia \\
\hline $7^{\mathrm{a}}$ & 10 minutos & $500-2$ vezes ao dia \\
\hline $8^{\mathrm{a}}$ & 15 minutos & 1000 - 1 vez ao dia \\
\hline $9^{a}$ & 15 minutos & $1200-1$ vez ao dia \\
\hline $10^{\mathrm{a}}$ & 20 minutos & $1600-1$ vez ao dia \\
\hline $11^{\mathrm{a}}$ & 20 minutos & 2000 - 1 vez ao dia \\
\hline $12^{\mathrm{a}}$ & 30 minutos & $3000-1$ vez ao dia \\
\hline
\end{tabular}

\section{Retorno ao trabalho}

Recomenda-se o retorno ao trabalho em torno de um a dois meses após o infarto e conforme orientação médica. Não é aconselhável viajar nos primeiros dois meses após o IAM. Também se ressalta que se evite viagens longas e cansativas ${ }^{1}$.

\section{Recomendações}

Durante a realização de qualquer atividade física, se ocorrer fadiga, mal estar, dor torácica, falta 
10.18606/2318-1419/amazonia.sci.health.v6n1p12-21 Revista Amazônia Science \& Health.

2018 Jan/Mar
Silva IM, Silva MG.

Infarto Agudo do Miocardio: Assitência ao paciente pós-infarto internado em Unidade de Terapia Intensiva. de ar e palpitação, recomenda-se que se interrompa a atividade imediatamente e procure um serviço de saúde especializado. É importante que se faça o uso correto das medicações prescritas e que o paciente compareça aos retornos médicos periódicos ${ }^{1}$.

\section{Prognóstico do paciente com IAM}

Estudos em países pioneiros que contam com times de resposta rápida (TRR), como, por exemplo, a Inglaterra e a Austrália, demonstraram que a identificação precoce de sinais de instabilidade clínica e a atuação das equipes de resposta rápida no atendimento de pacientes instáveis podem levar a diminuição no número de paradas cardíacas e de transferências não antecipadas para leitos de unidades de terapia intensiva (UTI), reduzindo a mortalidade dos pacientes hospitalizados ${ }^{23}$.

De acordo com Terzi ${ }^{24}$, vários sistemas de índices prognósticos têm sido desenvolvidos para avaliar a gravidade dos pacientes e estimar a mortalidade em Unidades de Terapia Intensiva (UTI). Sua aplicação procura identificar os pacientes com maior potencial de recuperação e que, portanto, beneficiar-se dos cuidados da UTI, facilitando a seleção daqueles que atenderão critérios de internação e alta.

A hiperglicemia da admissão de pacientes com síndrome coronariana aguda (SCA) é uma situação freqüente e constitui excelente preditor de sobrevida e complicações hospitalares em pacientes com ou sem diabetes melitos. A hiperglicemia no IAM está associada ao aumento de ácidos graxos livres, que induz arritmias cardíacas e resistência insulínica, levando a aumento de consumo de oxigênio e a piora da isquemia. A hiperglicemia também está relacionada com disfunção microvascular, estado pró-trombótico, inflamação vascular, além de disfunção endotelial ${ }^{25}$.

Nos estudos dos pacientes críticos com controle rigoroso da glicemia, verificou-se melhor evolução no grupo de pacientes que mantinham a glicemia entre 80 e $110 \mathrm{mg} / \mathrm{dl}$ nos pacientes em UTI cirúrgica. Entretanto, em um estudo subseqüente, observou-se que o controle rigoroso da glicemia não trazia benefício em pacientes que ficavam internados até três dias na UTI ou naqueles que tinham diagnóstico prévio de diabetes ${ }^{25}$.

Dentre os sistemas propostos, o mais utilizado atualmente em diversos países é o APACHE (Acute Physiology and Chronic Health Evaluation), desenvolvido inicialmente por Knaus, em 1981, e aprimorado, em 1985, para o APACHE II2. Outro sistema existente é o SAPS (Simplified Acute Physiology Score), que difere do APACHE II por conter outras variáveis clínicas, como diurese, bicarbonato e bilirrubina séricos, e por não considerar o diagnóstico de internação. Existem outros diferentes sistemas disponíveis, que têm aplicação para subgrupos específicos, como trauma, instabilidade hemodinâmica, isquemia miocárdica, pós-operatório de cirurgia cardíaca, disfunção orgânica múltipla (SOFA) e SEPSE, embora, no momento, poucos estudos estejam sendo desenvolvidos com o subgrupo de cardiopatas com insuficiência cardíaca (IC) ${ }^{26}$.

Em 1967, algumas classificações, relacionadas com a gravidade de pacientes com infarto agudo do miocárdio, começaram a ser descritas, como a classificação de KILLIP e, posteriormente, a classificação hemodinâmica do infarto agudo do miocárdio. Para outras patologias, como por exemplo, o trauma crânio-encefálico, a escala de coma de Glasgowé amplamente utilizada. O primeiro índice prognóstico proposto para pacientes internados em terapia intensiva foi 0 APACHE (Acute Physiology and Chronic Health Evaluation), desenvolvido por Knaus (Knaus e cols.) nos EUA, posteriormente aprimorado para a versão APACHE II2. Hoje é o sistema mais utilizado entre os índices de gravidade. Mais recentemente, foi introduzido o APACHE III, que utiliza dezessete variáveis, número superior às variáveis utilizadas na versão anterior. Embora a presença de arritmias, a hiponatremia, 0 tamanho das cavidades ventriculares e o nível de catecolaminas séricas sejam fatores comprovadamente importantes na evolução a longo prazo do cardiopata,estas variáveis também podem influenciar a fase aguda de descompensação. Nesta casuística, observamos que a hiponatremia, com sódio sérico inferior a $129 \mathrm{~m} \mathrm{Eq/l}$, dobra a mortalidade dos pacientes, em relação aos pacientes com dosagem de sódio normal $^{26}$.

Complicações do período pós-operatório foram definidas como: sangramento torácico significativo (acima de $500 \mathrm{~mL}$ por dia), re-operação, tempo prolongado de ventilação mecânica (acima de 12 horas), pneumotórax, derrame pleural volumoso (superior a $500 \mathrm{~mL}$ estimado por exame de imagem e/ou que necessitou toracocentese e/ou drenagem), fibrilação ou flutter atrial, disritmias ventriculares, IAM, insuficiência arterial periférica, infecções nosocomiais (presentes após 48 horas de hospitalização: pneumonia, infecção do trato urinário, bacteremia oriunda de cateter vascular, infecção de ferida esternal, mediastinite), novo AVE, crise convulsiva tônica-clônica, disfunção renal aguda (definida como aumento da creatinina maior que $50 \%$ ou que $2,0 \mathrm{mg} / \mathrm{dL}$ ou diurese menor que $0,5 \mathrm{~mL} / \mathrm{kg} /$ hora e necessidade de diuréticos) e morte durante hospitalização ${ }^{15}$.

\section{RESULTADOS E DISCUSSAO}

Na PubMed foram encontrado 151 artigos e na SciELO 126, totalizando 277 artigos. Estes artigos foram triados e excluídos os que não 
10.18606/2318-1419/amazonia.sci.health.v6n1p12-21 Revista Amazônia Science \& Health. 2018 Jan/Mar
Silva IM, Silva MG.

Infarto Agudo do Miocardio: Assitência ao paciente pós-infarto internado em Unidade de Terapia Intensiva. tratavam sobre assistência, restando 26 artigos que comporam esse estudo.

Os artigos selecionados foram analisados e classificados em 09 (nove) tópicos, sendo: 1) perfil do paciente com infarto agudo do miocárdio, 2) principais fatores de risco para infarto agudo do miocárdio; 3) epidemiologia do iam; intervenção no infarto agudo do miocárdio; 4) suporte ventilatório ao paciente com infarto agudo do miocárdio; 5) terapêutica adjuvante no infarto agudo do miocárdio; 6) eventos adversos da assistência em unidade de terapia intensiva; 7) assistência em enfermagem no infarto agudo do miocárdio; 8) manual de orientações com infarto agudo do miocárdio; 9) prognóstico do paciente com infarto agudo do miocárdio.

A maioria das experiências relatadas foi desenvolvida em países da América do Norte e Europa e as descrições de experiências em países em desenvolvimento, como o Brasil, são escassas.

A maioria da incidência de infarto agudo do miocárdio ocorreu nos indivíduos do sexo masculino, na proporção de 2/1. A literatura, ao discutir o papel do fator sexo no desenvolvimento da doença isquêmica cardíaca, comumente 0 faz relacionando-o com a idade. Tal fato é decorrente das características peculiares a cada sexo e que fazem homens e mulheres envelhecerem de maneiras distintas ${ }^{5}$.

Ao compararmos as incidências entre os dois sexos constatamos que a maior diferença entre e I as ocorreu na faixa dos 30-40 anos, 5 homens por 1 mulher. Com o avançar da idade esta diferença diminuiu, porém, em todas as faixas etárias, a incidência no sexo masculino foi maior (Tabela 2).

\section{Tabela 2. Perfil do paciente com IAM}

\begin{tabular}{ccccccc}
\hline Faixa Etária & Homens & Mulheres & \multicolumn{2}{c}{ Total } \\
\hline & $\mathrm{n}$ & $\%$ & $\mathrm{n}$ & $\%$ & $\mathrm{n}$ & $\%$ \\
$\mathbf{3 0 I}-\mathbf{4 0}$ & 4 & 8,9 & 1 & 2,2 & 5 & 11,1 \\
$\mathbf{4 0 I}-\mathbf{5 0}$ & 4 & 8,9 & 2 & 4,4 & 6 & 13,2 \\
$\mathbf{5 0 I}-\mathbf{6 0}$ & 7 & 15,5 & 5 & 11,1 & 12 & 26,6 \\
$\mathbf{6 0 l}-\mathbf{7 0}$ & 8 & 17,8 & 3 & 6,6 & 11 & 24,2 \\
$\mathbf{7 0 I}-\mathbf{8 0}$ & 7 & 15,5 & 3 & 6,6 & 10 & 22,2 \\
$\mathbf{8 0 I}-$ & - & - & 1 & 2,2 & 2 & 2,2 \\
\hline \multicolumn{1}{l}{ Total } & $\mathbf{3 0}$ & $\mathbf{6 6 , 7}$ & $\mathbf{1 5}$ & $\mathbf{3 3 , 3}$ & $\mathbf{4 5}$ & $\mathbf{1 0 0 , 0}$ \\
\hline
\end{tabular}

Segundo Dantas ${ }^{5}$, observou-se que, com relação à idade, a maior concentração de pacientes está na faixa etária dos 50 -70, sendo seguidos por aqueles com idade entre 70 e 80 anos. Esses resultados confirmam que 0 infarto atinge, predominantemente, os indivíduos de meia idade e idosos.

\section{CONSIDERAÇOES FINAIS}

De acordo com este estudo de revisão bibliografica, pode-se considerar que, o IAM é uma patologia de facil diagnóstico, mas que se nao tratada a tempo tem um alto indice de morte nas primeiras horas de sintomas. A gravidade do IAM depende principalmente da extensao da regiao atingida e de todos os fatores de riscos associados. Assim será de suma importancia a mudança de hábitos de vida o mais precoce possivel, mudança essa que na maioria dos casos irão colaborar para um bom prognóstico e ainda diminuir os riscos de reinfarto.

Contudo cabe ressaltar que o atendimento de qualidade prestado ao paciente pós-IAM, é a melhor maneira de recupera-lo e torna-lo indepente de auxílio para sua sobre vida e que a qualidade de vida do paciente em reabilitação pós IAM, influencia relativamente em sua recuperação e não reincidencia de infarto.

\section{REFERENCIAS}

1. Martino MMF, Suedekum EWM, Cassol MJ, Iza MM. Manual de orientação para paciente infartado. Rev. Bras. Enferm. [Internet]. 1994 Set [citado 2016 Jul 20] ; 47(3): 304-313. Disponível em: http://www.scielo.br/scielo.php?script=sci arttext \&pid=S0034-71671994000300012\&Ing=pt.

2. Marcolino MS, Brant LCC, Araujo JG, Nascimento BR, Castro LRA, Martins P. Implantação da linha de cuidado do infarto agudo do miocárdio no município de Belo Horizonte. Arq. Bras. Cardiol. [Internet]. $2013 \mathrm{Abr}$ [citado 2016 Set 23]; $100 \quad$ (4): $\quad 307-314$. Disponível em: http://www.scielo.br/scielo.php?script=sci_arttext \&pid $=$ S0066-782X2013000400001\&lng=pt. Epub 02-Abr-2013.

3. Beccaria LMM, Pereira RAM, Contrin LM, Lobo SMA, Trajano DHL. Eventos adversos na assistência de enfermagem em uma unidade de terapia intensiva. Rev. Bras. Ter. intensiva [Internet]. 2009 Ago [citado 2016 Set 23]; 21(3): 276-282. Disponível em: http://www.scielo.br/scielo.php?script=sci_arttext \&pid=S0103-507X2009000300007\&lng=pt.

4. Pesaro AEP, Serrano JR. Carlos VN, Nicolau JC. Infarto agudo do miocárdio: síndrome coronariana aguda com supradesnível do segmento ST. Rev. Assoc. Med. Bras. [Internet]. $2004 \mathrm{Abr}$ [citado 2016 Set 23]; 50(2): 214-220. Disponível em: http://www.scielo.br/scielo.php?script=sci_arttext \&pid=S0104-42302004000200041\&lng=pt. 
10.18606/2318-1419/amazonia.sci.health.v6n1p12-21 Revista Amazônia Science \& Health. 2018 Jan/Mar
5. Dantas RAS, Aguilar OM. Infarted patient's profile concerning the "health area" model. Rev. Bras. Enferm. [Internet]. $1998 \mathrm{Dez}$ [citado 2016 Ago 05]; 51(4): 571-588. Disponível em: http://www.scielo.br/scielo.php?script=sci_arttext \&pid=S0034-71671998000400004\&lng=pt.

6. Mattos MA, Toledo DG, Mattos CE, Tura BR, Gadelha DNB, Siqueira FAG. Tendência temporal de letalidade hospitalar por infarto agudo do miocárdio: 1994-2003. Arq. Bras. Cardiol. [Internet]. 2005 Maio [citado 2016 Nov 20]; 84(5): 416-421. Disponível em:

http://www.scielo.br/scielo.php?script=sci_arttext \&pid=S0066-782X2005000500012\&lng=pt.

7. Silva MAD, Sousa AGMR, Schargodsky $H$. Fatores de risco para infarto do miocárdio no Brasil: estudo FRICAS. Arq. Bras. Cardiol. [Internet]. 1998 Nov [citado 2016 Jul 20]; 71(5): 667-675. Disponível em: http://www.scielo.br/scielo.php?script=sci_arttext \&pid=S0066-782X1998001100005\&lng=pt.

8. Santos ES, Minuzzo L, Pereira MP, Castillo MTC, Palácio MAG, Ramos RF. Registro de síndrome coronariana aguda em um centro de emergências em cardiologia. Arq. Bras. Cardiol. [Internet]. 2006 Nov [citado 2016 Nov 20]; 87(5): 597-602. Disponível em: http://www.scielo.br/scielo.php?script=sci_arttext \&pid=S0066-782X2006001800008\&Ing=pt.

9. Ribeiro ALP. Os dois Brasis e o tratamento do infarto agudo do miocárdio. Arq. Bras. Cardiol. [Internet]. 2009. Disponível em: http://www.scielo.br/scielo.php?script=sci_arttext \&pid=S0066-782X2009000800003\&lng=en.

10.Portela A, Linhares C, Bastos R, Paiva J. Intervenção coronária em hospital geral no Piauí. Arq. Bras. Cardiol. [Internet]. 1998 Out [citado 2016 Ago 15]; 71 (4): 591-594. Disponível em: http://www.scielo.br/scielo.php?script=sci_arttext \&pid=S0066-782X1998001000006\&lng=pt.

11.Piegas LS, Haddad N. Intervenção coronariana percutânea no Brasil: resultados do Sistema Único de Saúde. Arq. Bras. Cardiol. São Paulo, v. 96, n. 4, p. 317-324, abr. 2011. Disponível em http://www.scielo.br/scielo.php?script=sci_arttext \&pid=S0066782X2011000400010\&lng=pt\&nrm=iso.

12.Teno LAC, Castilho OT, Menardi AC, Racy CJ, Cozac EP, Greguolo C. Cirurgia de revascularização no infarto agudo do miocárdio. Rev Bras Cir Cardiovasc [Internet]. 1990 Abr [citado 2016 Nov 19] ; 5(1): 9-15. Disponível em:

http://www.scielo.br/scielo.php?script=sci_arttext \&pid=S0102-76381990000100002\&lng=pt.

13.Ladeira RT, Jatene FB, Monteiro R, Zucato SP, Baracioli LM, Hueb AC. Cirurgia de revascularização na fase aguda do infarto do miocárdio: análise dos fatores pré-operatórios preditores de mortalidade. Arq. Bras. Cardiol. [Internet]. 2006 Set [citado 2016 Nov 20] ; 87( 3 ): 254-259. Disponível em: http://www.scielo.br/scielo.php?script=sci_arttext \&pid=S0066-782X2006001600005\&lng=pt.

14.Vegni $R$, Almeida GF, Braga $F$, Freitas $M$, Drumond Luis Eduardo, Penna Guilherme et al . Complicações após cirurgia de revascularização miocárdica em pacientes idosos. Rev. Bras. Ter. intensiva [Internet]. 2008 Sep [cited 2016 Nov 20] ; 20( 3 ): 226-234. Available from: http://www.scielo.br/scielo.php?script=sci_arttext \&pid=S0103-507X2008000300004\&lng=en.

15.Lima ES, Cruz CG, Santos FC, Gomes NM, Bittencourt HS, Reis FJFB. Suporte ventilatório na capacidade funcional de pacientes com insuficiência cardíaca: estudo piloto. Arq. Bras. Cardiol. [Internet]. 2011 Mar [citado 2016 Set 23]; 96(3): 227-232. Disponível em: http://www.scielo.br/scielo.php?script=sci_arttext \&pid=S0066-782X2011000300009\&Ing=pt. Epub 07-Jan-2011.

16. Habib SS, Kurdi MI, Al AZ, Suriya MO. Níveis de PCR são maiores em pacientes com síndrome coronariana aguda e supradesnivelamento do segmento ST do que em pacientes sem supradesnivelamento do segmento ST. Arq. Bras. Cardiol. [Internet]. 2011 Jan [citado 2016 Set 23]; 96(1): 13. Disponível em: http://www.scielo.br/scielo.php?script=sci_arttext \&pid=S0066-782X2011000100004\&Ing = $\overline{\text { pt. }}$. Epub 03-Dez-2010.

17. Gualandro DM, Calderaro D, Yu PC, Marques AC, Caramelli B. Betabloqueadores e operações não cardíacas: o que muda após o estudo POISE?. Arq. Bras. Cardiol. [Internet]. 2009 Nov [citado 2016 Nov 20]; 93(5): e82-e84. Disponível em:

http://www.scielo.br/scielo.php?script=sci_arttext \&pid=S0066-782X2009001100024\&lng=pt.

18.Silva LK, Escosteguy CC, Machado CV. Metodologia para a estimativa de padrões de qualidade: o caso do infarto agudo do miocárdio. Cad. Saúde Pública [Internet]. Disponível em: 
http://www.scielo.br/scielo.php?script=sci_arttext \&pid=S0102-311X1996000600008\&lng=pt.

19.Avezum A.Terapêutica adjuvante no infarto agudo do miocárdio: recomendações baseadas em evidências. Arq. Bras. Cardiol. [Internet]. $1998 \mathrm{Jul}$ [citado 2016 Mai 10]; 71(1): 03-03. Disponível em: http://www.scielo.br/scielo.php?script=sci_arttext \&pid=S0066 782X1998000700002\&lng=pt.

20.Laizo A, Delgado FEF, Rocha GM. Complicações que aumentam o tempo de permanência na unidade de terapia intensiva na cirurgia cardíaca. Rev Bras Cir Cardiovasc [Internet]. 2010 Jun [citado 2016 Nov 20]; 25(2): 166-171. Disponível em:

http://www.scielo.br/scielo.php?script=sci_arttext \&pid=S0102-76382010000200007\&lng=pt.

21.Wehbe G, Galvão MC. Aplicação da Liderança situacional em enfermagem de emergência. Rev. bras. enferm. [Internet]. 2005 Feb [cited 2016 Nov 20]; 58(1):33-38. Available from: http://www.scielo.br/scielo.php?script=sci_arttext \&pid=S0034-71672005000100006\&lng=en.

22. Vieira AC, Bertoncello KCG, Girondi JBR, Nascimento ERP, Hammerschmidt KSA, Zeferinho MT. Percepção dos enfermeiros de emergência na utilização de um protocolo para avaliação da dor torácica. Texto contexto enferm. [Internet]. 2016 [citado 2016 Ago 05]; 25(1): $830014 . \quad$ Disponível em: http://www.scielo.br/scielo.php?script=sci_arttext \&pid=S0104-07072016000100326\&lng=pt. Epub 05-Abr-2016.

23.Taguti PSilva, Dotti AZ, Araujo KP, Pariz PS, Dias GF, Kauss IAM. Atuação do time de resposta rápida em hospital universitário no atendimento de código amarelo. Rev. Bras. Ter. intensiva [Internet]. 2013 June [cited 2016 Nov 20]; 25(2): 99-105. Disponível em:

http://www.scielo.br/scielo.php?script=sci_arttext \&pid=S0103-507X2013000200007\&lng=en.

24.Terzi CB, Lage SG, Dragosavac D, Terzi RG. Insuficiência cardíaca grave em unidade de terapia intensiva: existe um índice prognóstico ideal?. Arq. Bras. Cardiol. [Internet]. 2006 Set [citado 2016 Set 23]; 87(3): 344-351. Disponível em:

http://www.scielo.br/scielo.php?script=sci_arttext \&pid=S0066-782X2006001600018\&lng=pt.

25.Góis AFT. Glicemia no prognóstico da síndrome coronariana aguda. Arq Bras Endocrinol Metab [Internet]. [cited 2016 Nov 20]; 52(3): 429-430. Disponível em:

http://www.scielo.br/scielo.php?script=sci_arttext \&pid=S0004-27302008000300002\&lng=en.

26.Piegas LS, Timerman A, Feitosa GS, Nicolau JC, Mattos LAP, Andrade MD. V Diretriz da Sociedade Brasileira de Cardiologia sobre Tratamento do Infarto Agudo do Miocárdio com Supradesnível do Segmento ST. Arq. Bras. Cardiol. [Internet]. 2015 .Disponível em: http://www.scielo.br/scielo.php?script=sci_arttext \&pid=S0066782X2015003000001\&lng=pt. 